\title{
Results from the VLTI-PRIMA Fringe Tracking Testbed
}

\author{
Johannes Sahlmann ${ }^{a, b}$, Roberto Abuter ${ }^{a}$, Nicola Di Lieto ${ }^{a}$, Serge Ménardi ${ }^{a}$, \\ Françoise Delplancke ${ }^{a}$, Hendrik Bartko ${ }^{c}$, Frank Eisenhauer ${ }^{c}$, Samuel Lévêque ${ }^{a}$, \\ Oliver Pfuhl ${ }^{c}$, Nicolas Schuhler ${ }^{a}$, Gerard van Belle $^{a}$ and Gautam Vasisht ${ }^{a, d}$ \\ ${ }^{a}$ European Southern Observatory, Karl-Schwarzschildstrasse 2, 85748 Garching, Germany; \\ ${ }^{b}$ Observatoire de Genève, 51 Chemin Des Maillettes, 1290 Sauverny, Switzerland; \\ ${ }^{c}$ Max Planck Institut für Extraterrestrische Physik, Giessenbachstr., 85748 Garching,Germany; \\ ${ }^{d}$ JPL-Caltech, 4800 Oak Grove Dr., Pasadena CA 91109, USA
}

\begin{abstract}
The Fringe Sensor Unit (FSU) is the central element of the dual-feed facility PRIMA at the VLT Interferometer (VLTI). Two identical FSU fringe detectors deliver real-time estimates of phase delay, group delay and signalto-noise ratio for the two observed targets. They serve both as the scientific instrument for astrometry with PRIMA and as sensor for the fringe tracking system of the interferometer. Prior to its installation at the VLTI scheduled for mid-2008, the FSU is going through an extensive laboratory test phase. It is therefore embedded in a semi-realistic environment, involving a VLTI-like control system and a laser metrology. This allows us to probe the system response to atmospheric piston jitter, tip-tilt disturbances and higher order aberrations, as they are expected at the observatory. We report on the system test results, outline the optimisation of the calibration procedure and we evaluate the FSU fringe tracking performance under realistic conditions. Finally, we compare the obtained performances to the scientific and technical requirements.
\end{abstract}

Keywords: Stellar Interferometry, VLTI, Fringe Tracking, Astrometry

\section{INTRODUCTION}

The Phase Referenced Imaging and Micro-Arcsecond Astrometry (PRIMA ${ }^{1}$ ) system will be deployed at the $\mathrm{VLTI}^{2}$ starting in July 2008 and is supposed to obtain first fringes before the end of the year. Once operational, the PRIMA dual-beam facility increases the sensitivity of the VLTI, enables phase referenced imaging and high-accuracy narrow-angle astrometry. In astrometric mode, two objects are simultaneously observed with two Unit or Auxiliary Telescopes (UT or AT) and the two beam pairs are combined in the fringe detector, the FSU. PRIMA comprises an infrared laser metrology system that delivers the internal differential optical path difference (OPD) between the two observed objects. The metrology end-points are located at the telescopes and in the FSU beam-combiners. The FSU consists of FSUA and FSUB, each combining two beams originating from the primary object and the secondary object, respectively. One of the scientific goals of astrometry with PRIMA is the extrasolar planet search and characterisation, where a large program is being prepared. ${ }^{3}$ PRIMA will also offer the possibility of galactic and extragalactic interferometric observations. ${ }^{4,5}$ In imaging mode, PRIMA provides two-telescope off-axis fringe tracking and the phase reference for the present VLTI-instruments AMBER and MIDI.

The VLTI-PRIMA Fringe Tracking Testbed was conceived in 2005 in order to characterise and test the FSU, before its installation at the VLTI. In June 2006, the FSU in its initial configuration, ${ }^{6}$ built by an external contractor, was installed in the testbed and it will remain there until its shipment to the Paranal Observatory in June 2008. It is embedded into a VLTI-similar control environment involving a dozen of real-time computers and several workstations. In combination with the specific testbed equipment, ${ }^{7}$ the testing and operation of the FSU becomes possible. Individual sub-system verification and general system performance testing can be carried out. Eventually, the FSU can be calibrated and can perform fringe-tracking in realistic conditions in terms of piston, tip-tilt and higher order disturbances.

Further author information: (Send correspondence to J.S.)

J.S.: E-mail: jsahlman@eso.org, Telephone: +49 8932006224 


\section{HARDWARE}

\subsection{Description of the FSU hardware}

While the detection principle of the FSU remained unchanged, fundamental modifications of the FSU with respect to its initial configuration ${ }^{6}$ were implemented in the testbed. A detailed description of the FSU and part of these modifications have previously been reported. ${ }^{8}$

The FSU operates in the infrared K-band and the science beams are combined in bulk optics, where a spatial phase modulation is introduced. Four beams with a relative phase separation of $\frac{1}{2} \pi$ provide the ABCD signals, used to reconstruct phase and group delay. Single-mode fibres spatially filter the beams and route the light to the cold camera. The cold camera was found unsatisfactory and was replaced during the testbed period. In the current design, the cryogenic infrared camera includes dispersive elements in order to obtain one white-light band and five spectral bands in each ABCD channel, hence a total number of 24 detector pixels are used (Figure $1)$. The phase delay $\phi(\mathrm{PD})$ is computed from the white-light band, while the group delay (GD) is estimated using the spectral bands. The flux ratio between the white-light band and the spectral bands is adjustable. In addition, white-light and spectral pixels can be read at different rates, which makes high-bandwidth PD measurements and low-bandwidth GD estimates with reduced noise available. Typically, the white-light band will be read at high-rate for accurate PD fringe tracking, whereas the spectral bands will be read at a lower rate and the resulting GD signal is used for fringe centering and the fringe search. This new cold camera configuration was implemented for both FSUA and FSUB.

Another important improvement was the replacement of the unstable initial FSU beam alignment unit with a system based on piezo-driven tip-tilt platforms, similar to the proven one of FINITO. ${ }^{9}$ The new FSU beam alignment system is simple, exhibits superior stiffness and most importantly allows for fast tip-tilt beam control. This functionality is essential for fringe tracking operation at the VLTI, since it makes for automated injection optimisation (beam tracking) and feed forward correction of real-time tip-tilt measurements (IRIS ${ }^{10}$ fast guiding $)^{8,11}$ possible. The read-out noise of the FSU detectors was reduced by implementing the Non-Destructive Read-Out mode, instead of the initial Correlated Double Sampling mode. The real-time software for phase and group delay computation was re-designed and significantly improved, including a new group delay algorithm. Finally, a temperature monitoring system for the FSU cryostat and cold camera was designed and implemented.

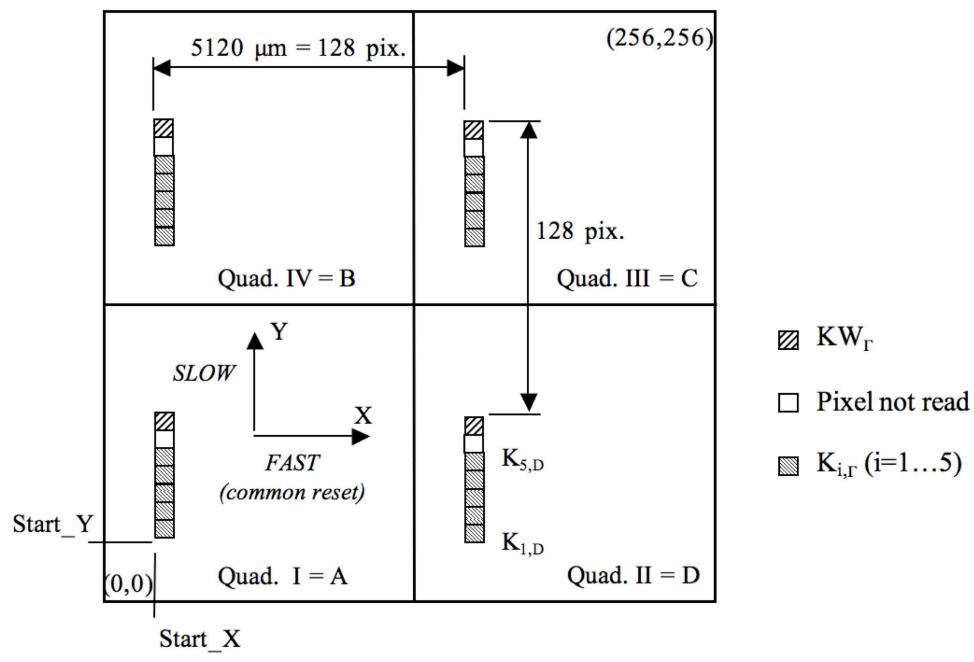

Figure 1. Sketch of the PICNIC detector layout for the FSU. The white-light pixels KW and the spectral pixels $\mathrm{K}_{i}$ are indicated (see Section 3.2 for the nomenclature and also Section 4.2).

\subsection{PRIMA metrology hardware}

There is a strong physical and operational link between the PRIMA metrology system ${ }^{1}$ and the FSU. Therefore, the metrology system was integrated, tested and validated in the testbed. The infrared laser beams $(\lambda=1319 \mathrm{~nm})$ 
with a diameter of $1 \mathrm{~mm}$ are injected through the FSU beam-combiner, follow the path of the stellar beams and propagate in the central obscuration. In the testbed they are retro-reflected by the beam generator.

The metrology hardware includes the laser source and the custom frequency-stabilisation system, ${ }^{12}$ the injection and extraction optics and three real-time computers.

\subsection{Description of the testbed hardware and the beam generator}

The testbed hardware includes a beam generator, delay line simulators and phase screens. ${ }^{7}$ The fibre-fed beam generator provides four coherent near-infrared ( $\mathrm{J}-, \mathrm{H}-$ and $\mathrm{K}$-band) input beams to the FSU and will replace the current internal calibration source of the VLTI. For the purpose of emulating the VLTI main delay lines, three delay line simulators are available. The optical path length can be modulated by a mirror mounted on a piezo-driven linear translation stage and the closed loop bandwidth of this system is $(500 \pm 10) \mathrm{Hz}$, which makes it suitable as fringe tracking actuator. For simulation of residual wavefront aberrations originating from the VLT UT Adaptive Optics system, rotating phase screens can be introduced in the beam paths.

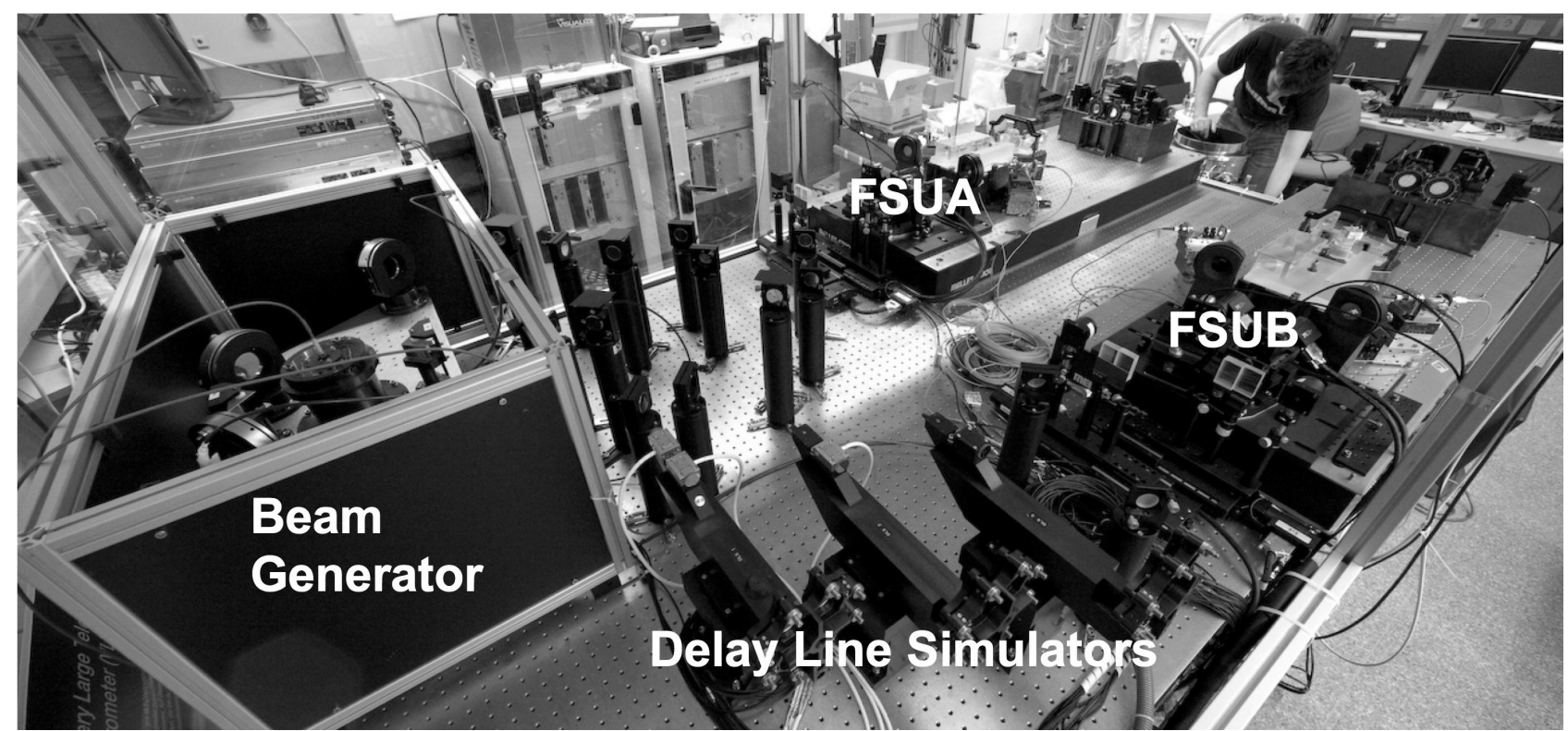

Figure 2. The testbed optical table with the beam generator, delay line simulators and the fringe sensors (ESO photo).

\section{SOFTWARE}

\subsection{Testbed environment and real-time computers}

The testbed computer environment is in accordance with ESO standards. Real-time computation and control at $\mathrm{kHz}$-data rates is performed by Local Control Units (LCUs), whereas high-level coordination and bookkeeping is done with Linux workstations. A number of 11 real-time computers and four workstations are installed in the testbed. All LCUs use the Reflective Memory Network (RMN) for fast communication. For compatibility with the VLTI, an RMN-architecture with two rings was implemented. The slower ring (General Electric (GE) 5576 cards) is used to interface the existing VLTI RMN, whereas the faster ring (GE 5565 cards) comprises the PRIMA LCUs. All LCUs are time-synchronised at $\mu$ s-level with the ESO fibre-optics system. The LCUs controlling FSUA, FSUB and the metrology system depend on the PRIMA workstation and will be installed at the VLTI. The acquisition computers implement the FSU real-time algorithms and the alignment computers control the actuators of the alignment system. The internal OPD computation and the laser frequency stabilisation are handled by three metrology computers.

Clones of existing VLTI-computers emulate the interferometer control environment. An OPD controller LCU is used for fringe tracking and an additional testbed computer controls the delay line simulators. We use filters to 
match the transfer functions of VLTI delay lines and simulators. ${ }^{7}$

The RMN recording facility ${ }^{13}$ was developed and implemented in the testbed. It allows to record virtually all data available on the RMN in real-time and has also been successfully implemented for the VLTI. The instrument calibration procedures were developed with ESO templates running on the instrument workstation.

\subsection{Real-time algorithms}

The FSU real-time algorithms for phase delay, group delay and signal-to-noise ratio (SNR) are evaluated based on the 24 instantaneous detector counts $I_{i, \Gamma}(t)$ at time $t$, where $i \in\{0,1,2,3,4,5\}$ denotes the spectral band and $\Gamma \in\{A, B, C, D\}$ denotes the ABCD-channel. $i=0$ stands for the white-light band and $i=1, . ., 5$ stands for the five spectral bands. The typical sampling rate and hence execution rate is $1 \mathrm{kHz}$. A number of parameters have to be pre-computed during the calibration and stored in the FSU database, where they can be accessed from the real-time computers. These calibration parameters are:

1. Dark: One bias value $G_{i, \Gamma}$ for each pixel used for dark-correction.

2. Flat: One flat value $F_{i, \Gamma}$ for each pixel used for photometric correction.

3. Wavelength: One effective wavelength value $\lambda_{i, \Gamma}$ for each pixel.

4. Phase-shift error coefficients: 6 values $\alpha_{i}, \beta_{i}, \gamma_{i}$ and $\delta_{i}$ for each ABCD-channel. The phase shift errors $\phi_{i, B}, \phi_{i, C}$ and $\phi_{i, D}$ are defined as the deviation of the B,C and D channel phase-shifts from their nominal values of $\frac{1}{2} \pi, \pi$ and $\frac{3}{2} \pi$, respectively. Then

$$
\alpha_{i}=\sin \phi_{i, C}, \beta_{i}=1+\cos \phi_{i, C}, \gamma_{i}=\cos \phi_{i, B}+\cos \phi_{i, D} \text { and } \delta_{i}=-\sin \phi_{i, B}-\sin \phi_{i, D} .
$$

5. Visibility noise: One value $v_{i}$ for each spectral band used to compute the SNR.

The current algorithms do not take the individual per-pixel wavelengths into account, but the mean wavelength $\lambda_{i}$ and wavenumber $\sigma_{i}$ in each spectral band:

$$
\lambda_{i}=\frac{1}{4} \sum_{\Gamma} \lambda_{i, \Gamma}, \quad \sigma_{i}=\frac{1}{\lambda_{i}} .
$$

The raw detector counts are dark-corrected and normalised with the photometric factor to yield the signals $S_{i, \Gamma}$ :

$$
S_{i, \Gamma}(t)=\frac{I_{i, \Gamma}(t)-G_{i, \Gamma}}{F_{i, \Gamma}}
$$

Real and imaginary part of the fringe phasors for each spectral band are computed from these signals:

$$
\begin{aligned}
X_{i}(t) & =\left(S_{i, A}(t)-S_{i, C}(t)\right) \gamma_{i}-\left(S_{i, B}(t)-S_{i, D}(t)\right) \alpha_{i}, \\
Y_{i}(t) & =\left(S_{i, B}(t)-S_{i, D}(t)\right) \beta_{i}-\left(S_{i, A}(t)-S_{i, C}(t)\right) \delta_{i} .
\end{aligned}
$$

The phase delay $\phi$ is computed from the white-light band with a non-ambiguity range of one wavelength $\lambda_{0}:{ }^{14}$

$$
\tan \phi(t)=\frac{X_{0}(t)}{Y_{0}(t)}
$$

To estimate the group delay, we calculate the Discrete Fourier Transformation (DFT) $\mathcal{F}$ of the fringe phasors

$$
\mathcal{F}(x, t)=\sum_{i=1}^{5}\left(X_{i}(t)+j Y_{i}(t)\right) \mathrm{e}^{-j 2 \pi \sigma_{i} x},
$$

where $j=\sqrt{-1}$ and $x$ spans an equally spaced range of $\pm 12 \mu \mathrm{m}$ with 60 points, i.e. $x=x_{1}, . ., x_{N}$ with $N=60$, $x_{1}=-12 \mu \mathrm{m}$ and $x_{60}=12 \mu \mathrm{m}$. A first estimate of the group delay is the displacement $x_{M}$ for which the DFT modulus is maximum:

$$
\mathrm{GD}(t)=x_{M} \quad \text { such that } \quad\left|\mathcal{F}\left(x_{M}, t\right)\right| \geq|\mathcal{F}(x, t)| \forall x .
$$


To refine this estimate, we compute the final GD value from the maximum of the parabolic fit to $\left|\mathcal{F}\left(x_{M}, t\right)\right|$ and two neighbouring values $\left|\mathcal{F}\left(x_{M \pm 1}, t\right)\right|$. Consequently, the FSU delivers GD estimates over a range of $\pm 12 \mu \mathrm{m}$ across the central fringe (Figure 6 ). Finally, the SNR is computed from the squared fringe amplitude $\eta_{i}^{2}(t)$ and the visibility noise $v_{i}$ given from the calibration:

$$
\begin{gathered}
\eta_{i}^{2}(t)=\left[\left(S_{i, A}(t)-S_{i, C}(t)\right) \beta_{i}-\left(S_{i, B}(t)-S_{i, D}(t)\right) \delta_{i}\right]^{2}+\left[\left(S_{i, B}(t)-S_{i, D}(t)\right) \gamma_{i}-\left(S_{i, A}(t)-S_{i, C}(t)\right) \alpha_{i}\right]^{2}, \\
\operatorname{SNR}_{i}(t)=\frac{\eta_{i}(t)}{v_{i}} .
\end{gathered}
$$

The state machine of the OPD controller relies on the $\mathrm{SNR}_{0}(t)$ in the white-light band.

\section{TESTING AND RESULTS}

\subsection{Calibration procedure}

The FSU calibration procedure was developed in the testbed. It relies on the internal metrology and makes use of the RMN recording facility. Currently, the procedure is composed of an automated part, implemented with instrument templates, followed by additional data processing, which requires minor manual intervention. The calibration principle has already been reported, ${ }^{8,14}$ however it was applicable to the initial FSU configuration. Here we describe the procedure for the laboratory calibration in the current configuration. It is also applicable for on-sky calibration, but becomes more involved due to the implication of telescopes, delay lines and other VLTI-systems.

FSUA and FSUB need to be calibrated independently and the procedure is identical in either case. This requirement was discovered in the testbed and gave rise to an important upgrade of the internal metrology system: we implemented an independent metrology for FSUB in order to retrieve the internal OPD of FSUA and FSUB individually. This comprised the development of a new architecture of the metrology real-time computers.

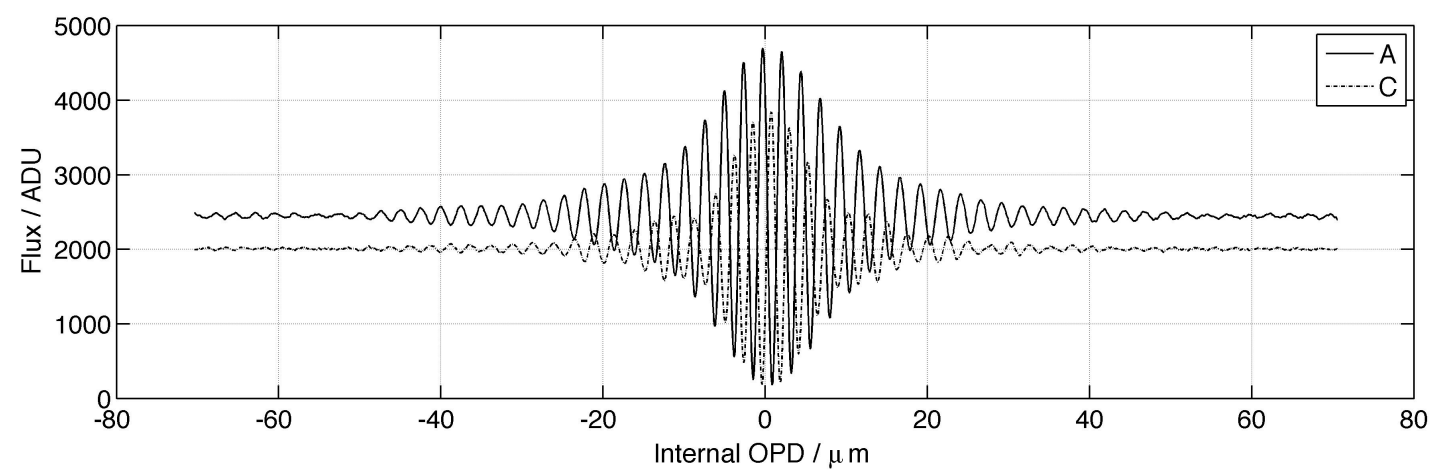

Figure 3. Combined white-light fringe packets in channel $\mathrm{A}$ and $\mathrm{C}$ from a calibration run. Note the opposite phase of the two signals, due to the spatial phase modulation.

\subsubsection{Dark calibration}

The bias values $G_{i, \Gamma}$ for the dark-correction are computed from a 20 s exposure of the detector signals $G_{i, \Gamma}(t)$ in the 24 pixels, where both incoming beams are blocked by shutters in the FSU alignment system. The darkcalibration template computes the mean values $G_{i, \Gamma}=\left\langle G_{i, \Gamma}(t)\right\rangle$ and updates the database entries accordingly.

\subsubsection{Flat calibration}

The photometric flat-signals $F_{i, \Gamma}$ are obtained in two steps. First Beam2 is blocked and a $20 \mathrm{~s}$ exposure of the 24 pixels is taken, yielding the flat signals $F 1_{i, \Gamma}(t)$ of Beam1. The analogous procedure provides the flat signals $F 2_{i, \Gamma}(t)$ of Beam2. The flat-calibration template updates the FSU database with the dark-corrected flat values $F_{i, \Gamma}=\left(\left\langle F 1_{i, \Gamma}(t)\right\rangle-G_{i, \Gamma}\right)+\left(\left\langle F 2_{i, \Gamma}(t)\right\rangle-G_{i, \Gamma}\right)$, where the brackets denote time averaging. 


\subsubsection{Fringe calibration}

The computation of phase delay, group delay and SNR require the precise values of the effective wavelength and phase shift error of each pixel and the visibility noise in each wavelength band. These parameters are deduced from the fringe calibration. We use Fourier Transform Spectroscopy to derive the effective wavelengths. Several OPD scans over the white-light fringe packet are performed with a linear motor, while the FSU detector counts and the internal OPD (measured with the internal metrology system) are recorded simultaneously. For each of the 24 channels, we combine the consecutive scans based on the metrology reference, see Figure 3 . The fringes are smoothed and interpolated for equal spacing before we compute the effective wavelengths $\lambda_{i, \Gamma}$ from the barycentre of the Fourier Transform modulus (Figure 4). The default calibration parameters that are used in the testbed are a number of 4 fringe scans and an effective scanning range of $140 \mu \mathrm{m}$. This approximately leads to 50 samples per fringe and a wavelength resolution of $32 \mathrm{~nm}$. The relative phase shifts of the ABCD-channels are derived by cross-correlating their fringe packets. Offsets of the crosscorrelation-functions with respect to the autocorrelation of channel $\mathrm{A}$ are converted into phase based on the effective wavelengths. The resolution here is $1 \mathrm{mrad}$. Eventually, the phase-shift error coefficients $\alpha_{i}, \beta_{i}, \gamma_{i}$ and $\delta_{i}$ are derived. We measure large deviations of the phaseshifts from the nominal values of $\frac{1}{2} \pi, \pi$ and $\frac{3}{2} \pi$, which can reach $\frac{1}{4} \pi$ in extreme cases and a slight chromatic dependence. The reasons for these large deviations are not clearly understood, but must be caused by imperfections in the FSU optical components.

Once the wavelengths and phase-shift errors are known, the visibility noise $v_{i}$ or equally the fringe amplitude in absence of fringes can be computed from the dark corrected flat exposure $\hat{F}_{i, \Gamma}(t)$ :

$$
\begin{gathered}
\hat{F}_{i, \Gamma}(t)=\left(F 1_{i, \Gamma}(t)-G_{i, \Gamma}\right)+\left(F 2_{i, \Gamma}(t)-G_{i, \Gamma}\right), \\
v_{i}^{2}=\left\langle\left[\left(\hat{F}_{i, A}(t)-\hat{F}_{i, C}(t)\right) \beta_{i}-\left(\hat{F}_{i, B}(t)-\hat{F}_{i, D}(t)\right) \delta_{i}\right]^{2}+\left[\left(\hat{F}_{i, B}(t)-\hat{F}_{i, D}(t)\right) \gamma_{i}-\left(\hat{F}_{i, A}(t)-\hat{F}_{i, C}(t)\right) \alpha_{i}\right]^{2}\right\rangle,
\end{gathered}
$$

where the brackets denote time averaging.

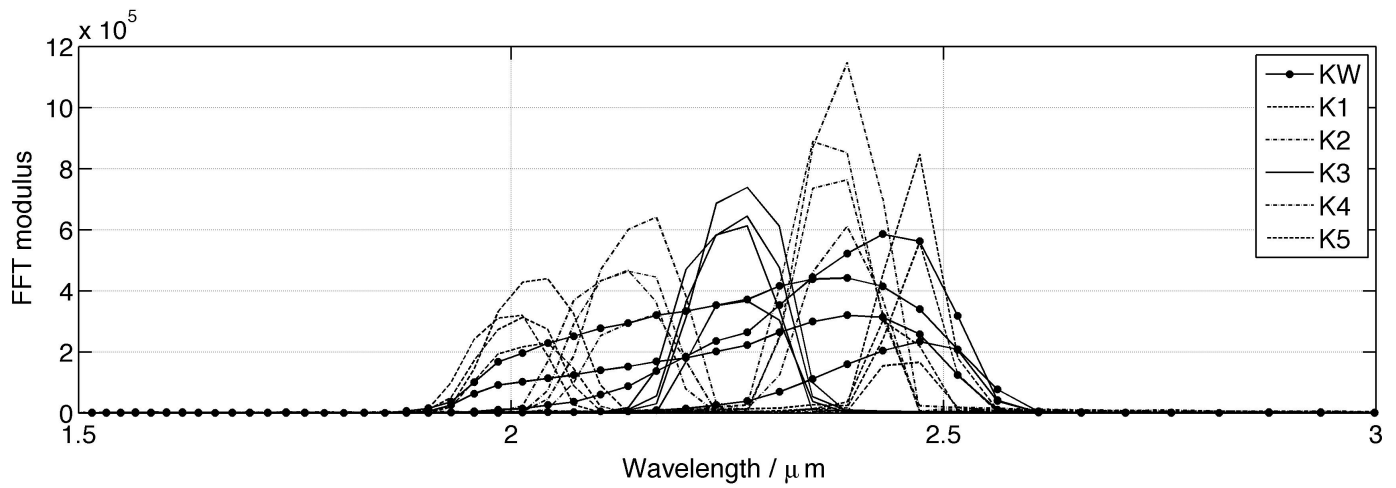

Figure 4. Fourier Transform modulus of all 24 channels from a calibration run. The wavelength variation over the spectrum channels K1..K5 and the broad white-light channels KW are visible.

\subsection{Detector Noise}

One key aspect for the observation of faint objects is the detector noise (see also next section). Each FSU uses one Teledyne 256x256 PICNIC detector with four simultaneously sampled 128x128 pixel quadrants, one quadrant per ABCD-channel. Only a subset of 6 pixels in one column per quadrant (Figure 1) is read, which reduces the read-out time compared to the full frame by a factor of $10^{3}$. The clocking rate, which is the time between two consecutive pixel reads, is $250 \mathrm{kHz}$. In the Correlated Double Sampling read mode the typical read-out noise is $\sim 30 e^{-}$. The read-out noise (RON) is reduced when using the Non-Destructive-Read-out (NDR) mode, where each pixel is sampled $N$ times during one integration time and the final detector count is computed from a linear fit to the $N$ sub-samples. The Poisson distributed RON decreases with roughly $1 / \sqrt{N}$, therefore $N$ shall be made as large as the detector integration time (DIT) allows. The typical FSU DIT of $1 \mathrm{~ms}$ allows for $N=16$ 
samples and the corresponding RON is $(19 \pm 1) e^{-}$. All FSU estimates are derived from count differences in two quadrants, see Eq. (4), hence we can define the pixel effective noise as the measured noise of the corresponding count difference divided by $\sqrt{2}$. The effective noise for FSUB is $(16 \pm 1) e^{-}$and lower than the individual RON, hence the quadrants are correlated in terms of noise.

Currently, the number of samples is limited by software to $N=16$ for $1 \mathrm{~ms}$ DIT at $250 \mathrm{kHz}$ clocking rate. We estimate the expected noise with larger $N$ from the discharge behaviour of individual pixels, see Figure 5 . The measured and the expected RON with $N=16$ for $1 \mathrm{~ms}$ DIT are in good agreement. The read-out speed and consequently $N$ can be increased with a modified NDR mode, where the pixel addressing is optimised and the overall clocking speed is set up to $1 \mathrm{MHz}$. Table 1 shows the maximum number of $N$ for different clocking rates and the expected noise. A clocking rate of $500 \mathrm{kHz}$ shows a similar noise behaviour, thus significant improvements in terms of size of $N$ and RON can be achieved. The current limitations, originating from the data-transfer and real-time computation load, can to some extend be overcome with optimised algorithms and faster computers.

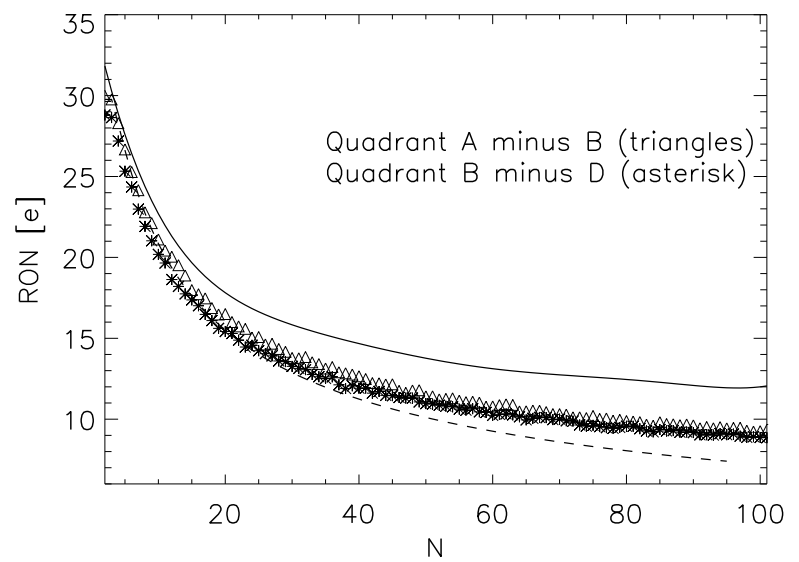

Figure 5. The pixel effective noise for quadrant differences as function of $\mathrm{N}$ for $1 \mathrm{~ms}$ DIT. A model of the individual RON (solid curve) and the theoretical limit (dashed curve) are drawn.

Table 1. The maximum number of $N$ in $1 \mathrm{~ms}$ with the optimised read mode as function of the clocking rate. The expected individual and effective RON are taken from Figure 5.

\begin{tabular}{|c|c|c|c|}
\hline Clocking rate & $N$ & individual RON / $e^{-}$ & effective RON / $e^{-}$ \\
\hline $250 \mathrm{kHz}$ & 22 & $17 \pm 1$ & $14 \pm 1$ \\
\hline $333 \mathrm{kHz}$ & 30 & $16 \pm 1$ & $13 \pm 1$ \\
\hline $500 \mathrm{kHz}$ & 46 & $14 \pm 1$ & $12 \pm 1$ \\
\hline $1 \mathrm{MHz}$ & 95 & $12 \pm 1$ & $9 \pm 1$ \\
\hline
\end{tabular}

\subsection{Linearity, bandwidth and noise of FSU estimates}

The linearity of phase and group delay estimates is essential to assure a reasonable control signal for the fringe tracking loop. Based on the internal metrology, we estimate the non-linearity from the deviation of the delay measurement slope from unity. The phase delay linearity is satisfactory, while the group delay linearity is worse than anticipated, which is due to the applied real-time algorithm that does not take into account the individual wavelengths of the ABCD-channels, but their mean value (Eq. (2)). However, we estimate the gain margin of the fringe tracking control loop to be large enough in order to cope with this non-linearity.

The 3dB-bandwidth of the phase delay estimate was measured to $(910 \pm 40) \mathrm{Hz}$. In addition, we identified the phase delay noise performance, which defines the FSU sensitivity and consequently the PRIMA fringe tracking limiting magnitude. During fringe tracking without external disturbance, we altered the detector integration time 
and the flux level independently, such that both photon-noise and readout-noise limited regimes were covered. While accounting for the control-loop sensitivity, we can estimate the PD noise from the PD power spectral density. For detector integration times of $0.5 \mathrm{~ms}, 1 \mathrm{~ms}$ and $2 \mathrm{~ms}$ with readout-noise of $(27 \pm 1) e^{-},(19 \pm 1) e^{-}$ and $(18 \pm 1) e^{-}$, respectively, we found the PD RMS noise to be $1.1-1.6$ times larger than the theoretical value. What remains to be done is to derive a model reproducing these measured values, such that the fringe tracking control loop parameters can be adapted to the atmospheric and photometric conditions during operation.
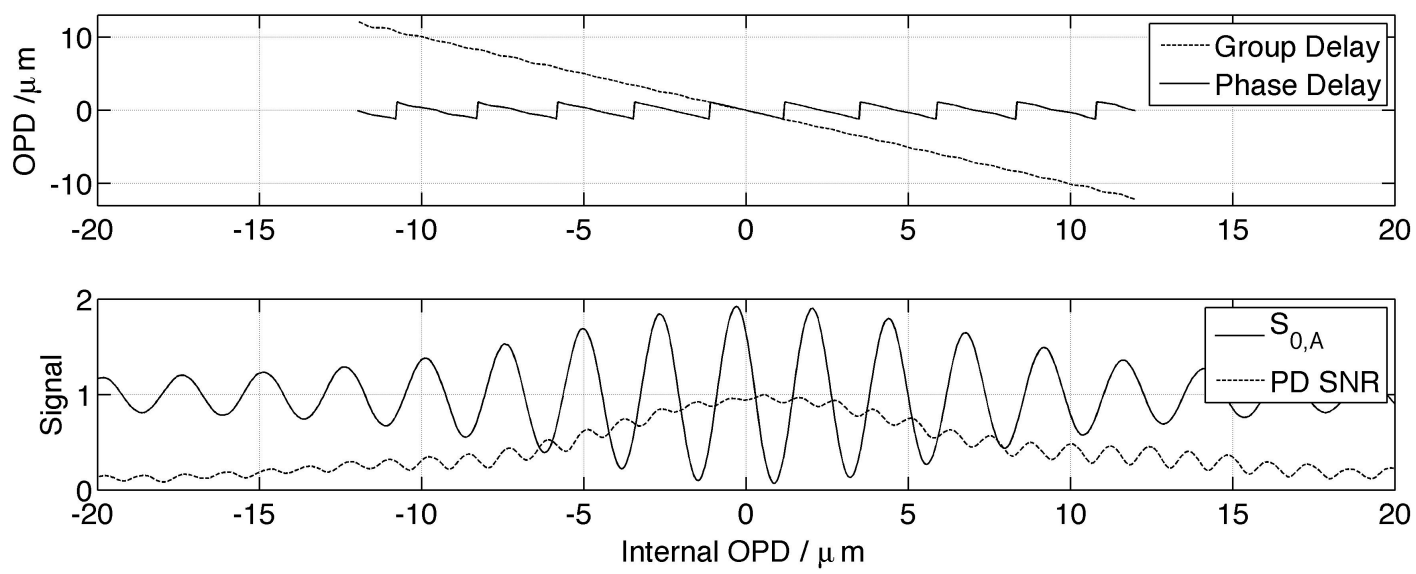

Figure 6. Phase delay and group delay estimates (top panel) and the phase delay SNR and one FSU signal (bottom panel) as function of internal OPD. GD is delivered over a range of $\pm 12 \mu \mathrm{m}$ and the PD is wrapping with a $\lambda_{0}$-period.

\subsection{FSU opto-mechanical stability}

While the overall stiffness of the FSU opto-mechanical components was found to be satisfactory, the longterm stability turned out to be problematic. In general, the thermal conditions in terms of long- and shortterm stability are worse in the testbed than in the Paranal VLTI laboratory. Hence, it is possible that the optomechanical stability of the FSU improves, once it is installed at the VLTI.

\subsubsection{Fibre motion causing differential injection}

Both FSUA and FSUB have four single-mode fibres that constitute the spatial filters for the ABCD-beams. ${ }^{6,8}$ These fibres are manually co-aligned using standard fibre-positioners. Thermal stress and mechanical instabilities cause drifts of the fibre positions. While common drifts can automatically be compensated with the FSU alignment system, relative drifts of one fibre with respect to another have to be corrected manually. Poor co-alignment of the ABCD-fibres causes differential injection effects, which severely limit the fringe tracking performance in presence of beam tip-tilt perturbations.

We monitored the ABCD-fibre positions over 34 days in the testbed and measured the relative motion to be $1.2-6.9 \mu \mathrm{rad}$ RMS. This corresponds to $2.4-13.8$ mas on the sky with an AT and thus to $1-6 \%$ of the theoretical FWHM of the Point Spread Function (PSF), which is 244 mas in K-band.

\subsubsection{Group Delay Bias stability}

A stringent requirement on the FSU long-term stability is set by the astrometric accuracy goal of PRIMA. The astrometric observable, yielding the angular separation of the two observed objects, is deduced from the group delay difference of FSUA and FSUB. In consequence, any group delay bias of the FSU which occurs on the time-scale of the observation (typically $30 \mathrm{~min}$ ) directly distorts the result of an astrometric measurement.

With the initial FSU configuration we measured the GD bias stability to be many times worse than the required level. This is linked to the initial design of the cold camera, which makes the GD measurement extremely sensitive to the thermal conditions inside and outside the cryostat. Temperature variations induce an image 
motion on the detector, which are interpreted as GD variations and cause the GD bias.

Thermal control of the cryostat vessel to a level of $0.05 \mathrm{~K}$ did not yield acceptable results. Better, but still not satisfactory, GD bias stability was achieved by thermally isolating the cryostat vessel from the ambient. Good stability was demonstrated for periods of hours, however these 'good' periods are not predictable during observation. In order to solve the GD stability problem, a new actuation system for the cold camera is currently under implementation in the testbed, involving cryogenic linear piezo actuators. Tests of this system are ongoing at the time of writing.

\subsection{Fringe Tracking}

We implemented a complete fringe tracking system in the testbed and included several possibilities to introduce perturbations into the control loop, see Figure 9.

\subsubsection{Injected disturbances}

The delay line simulator is used as actuator of the fringe tracking control loop and at the same time to inject piston disturbances into the beam path. Two types of piston perturbations are injected. First a model atmosphere which was generated in order to reproduce median seeing conditions in Paranal, with an amplitude of roughly $7 \mu \mathrm{m}$ OPD RMS. Second, we used FINITO to record the piston disturbance in Paranal during a technical VLTIrun on UT3 and UT4 in September 2006. In the testbed this disturbance sequence was played-back and also here the amplitude is roughly $7 \mu \mathrm{m}$ OPD RMS. Figure 7 shows the spectra of both piston disturbance types. The vibrations present in the VLTI when observing with the UTs are obvious.

The FSU alignment system uses one piezo tip-tilt platform per input beam. We use this fast tip-tilt actuator to introduce tip-tilt perturbations as they are present in the VLTI. Measurements of beam tip-tilt were recorded in November 2007 at the VLTI with an AT, using the IRIS tip-tilt sensor. These measurements represent the residuals of the AT tip-tilt correction system in addition to the tip-tilt introduced in the VLTI tunnel and light ducts. We produced a model that matches these measurements in terms of amplitude and spectrum and named it STRP. A second model, which includes the predicted IRIS Fast Guiding (IFG) open loop correction and where hence the low-frequency tip-tilt disturbances are rejected, is available and termed SIFG. The third model SIFG2 is the same as SIFG with half the amplitude. The resulting disturbance amplitudes amount to radial $23 \%$, $15 \%$ and $7 \%$ RMS of the PSF FWHM per beam for STRP, SIFG and SIFG2, respectively. Figure 8 shows the spectra of these three tip-tilt perturbations.

To simulate residual wavefront aberrations originating from the the VLT Unit Telescope Adaptive Optics system, we introduced rotating phase screens in the beam paths.

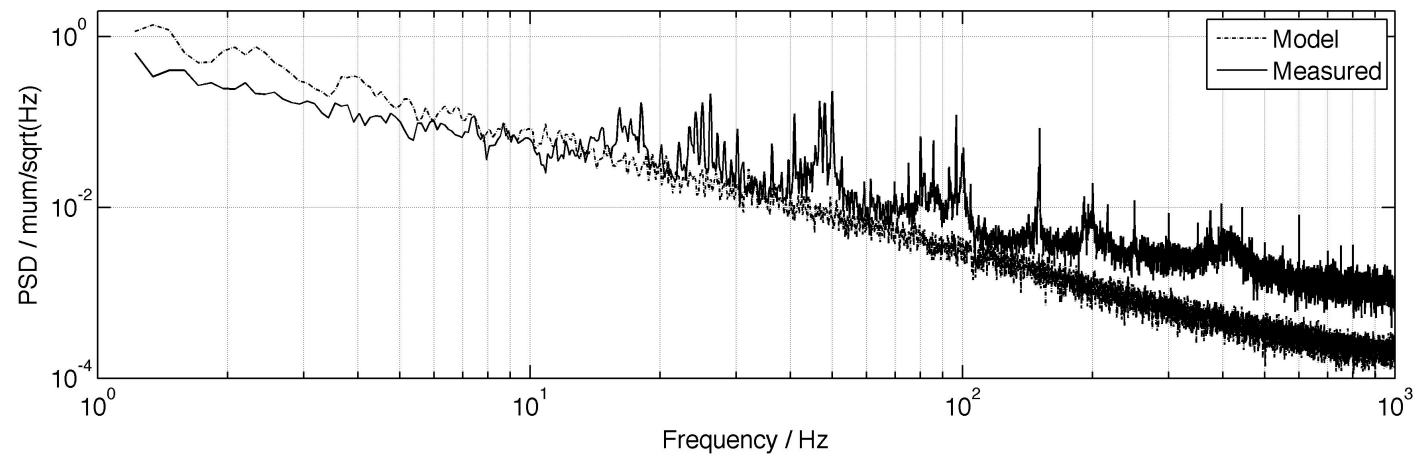

Figure 7. Spectra of the atmospheric piston model and of the piston disturbance measured with FINITO on 2 UTs.

\subsubsection{Setup and control algorithm}

We use a black body light source at $700^{\circ} \mathrm{C}$ to generate the light beams for FSU calibration and fringe-tracking. The typical FSU data rate is $1 \mathrm{kHz}$, while the OPD controller is running at $2 \mathrm{kHz}$ (like at the VLTI) and the metrology delivers internal OPD at a rate of $8 \mathrm{kHz}$. Figure 9 shows the layout of the fringe tracking control 


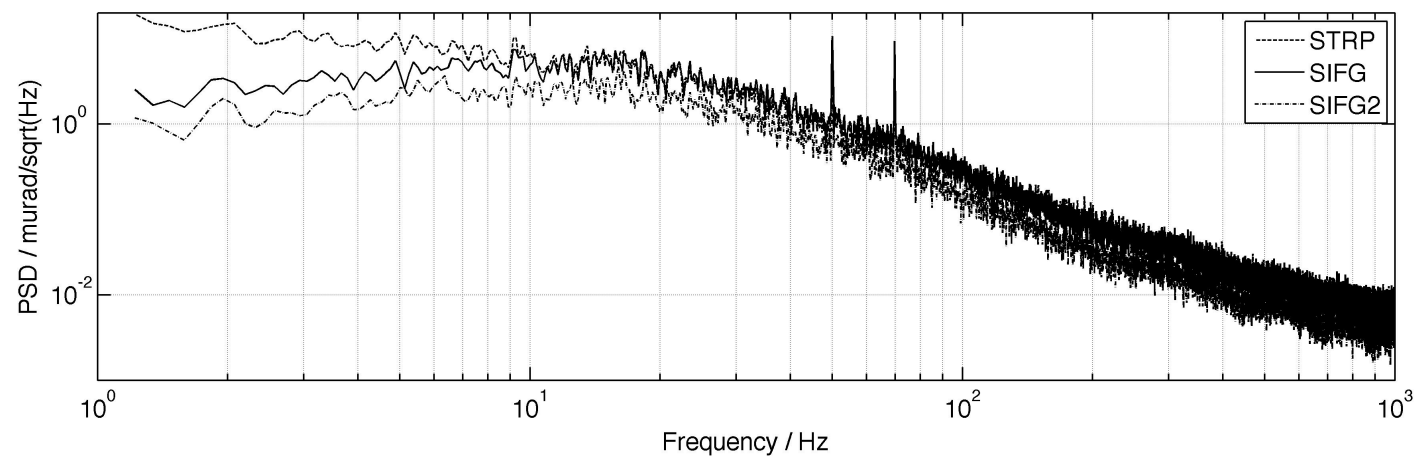

Figure 8. Tip-Tilt disturbance spectra for different models.

loop. In order to have a realistic actuator bandwidth, we use a filter that matches the transfer function of the testbed delay line simulators to a typical VLTI delay line, including its large actuation delay.

The control algorithm combines both slow group delay and fast phase delay tracking. Instead of controlling to zero-phase, the phase delay controller target is a variable designed to maintain the group delay signal around zero. This variable target depends on the integral of the group delay. The time constant of the group delay integral is of the order of a few seconds and large compared to $\sim 0.1 \mathrm{~s}$ for the phase controller. In consequence, the control algorithm is tracking the central fringe of maximum coherence.

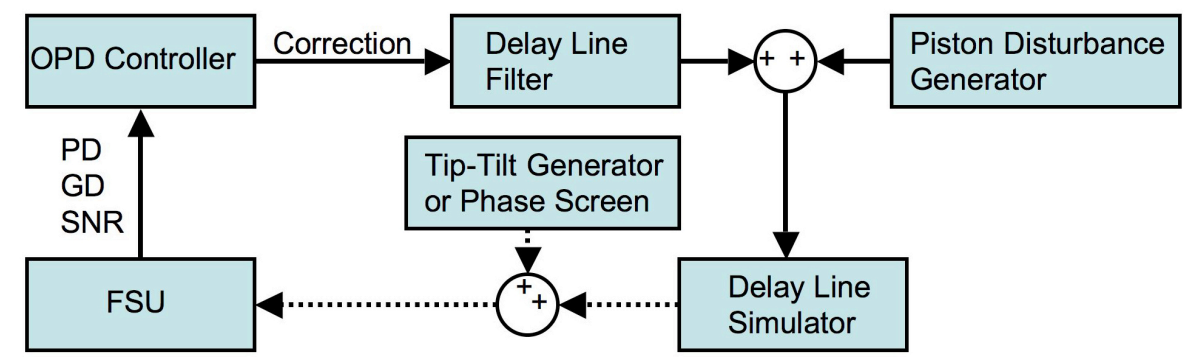

Figure 9. The testbed fringe tracking control system: PD, GD and SNR estimates are delivered to the OPD controller, which counteracts disturbances by generating real-time corrections. These are filtered to obtain a VLTI-similar actuator transfer function. Disturbance generators are included and the optical part of the loop is indicated with the dotted arrows.

\subsubsection{Fringe tracking results}

We achieved stable fringe-tracking with the FSU at high-flux in realistic conditions in terms of piston, tip-tilt and higher order disturbances, as they are described in Section 4.5.1. Based on the metrology measurement of the internal OPD, we can evaluate the tracking performance in terms of closed loop residual OPD. When injecting the atmospheric piston model, we measure the OPD residual to $(276 \pm 1) \mathrm{nm}$ RMS over $1 \mathrm{~s}$, see Figure 10. This value is almost doubled (97\% increase) when we inject the measured VLTI piston disturbance, which demonstrates the susceptibility of the control loop to UT-vibrations that occur in the frequency region where the controller rejection is low. ${ }^{15}$

In presence of tip-tilt disturbances and absence of piston disturbance, the OPD residuals are higher in closed loop than in open loop, hence fringe tracking is disadvantageous in these conditions. The increase from open to closed loop residual OPD amounts to $(43 \pm 1) \%,(25 \pm 2) \%$ and $(3 \pm 3) \%$ for the STRP, SIFG and SIFG2 tip-tilt disturbance, respectively. Hence this effect depends on the perturbation amplitude and it can be explained with differential injection effects (see also Section 4.4.1). Beam tip-tilt causes differential injection fluctuations in the ABCD-fibres, which are interpreted as phase delay jitter by the FSU. This apparent jitter is then re-injected into the fringe tracking loop and deteriorates its performance. 
By adding tip-tilt perturbations to the atmospheric piston model disturbance, the residuals are increased by $(15 \pm 4) \%$ and $(7 \pm 1) \%$ for STRP and SIFG model, respectively.

Finally, we found that the FSU is not very sensitive to higher order aberrations injected with the phase screens. The fringe tracking performance is dominated by the piston and tip-tilt disturbance conditions.

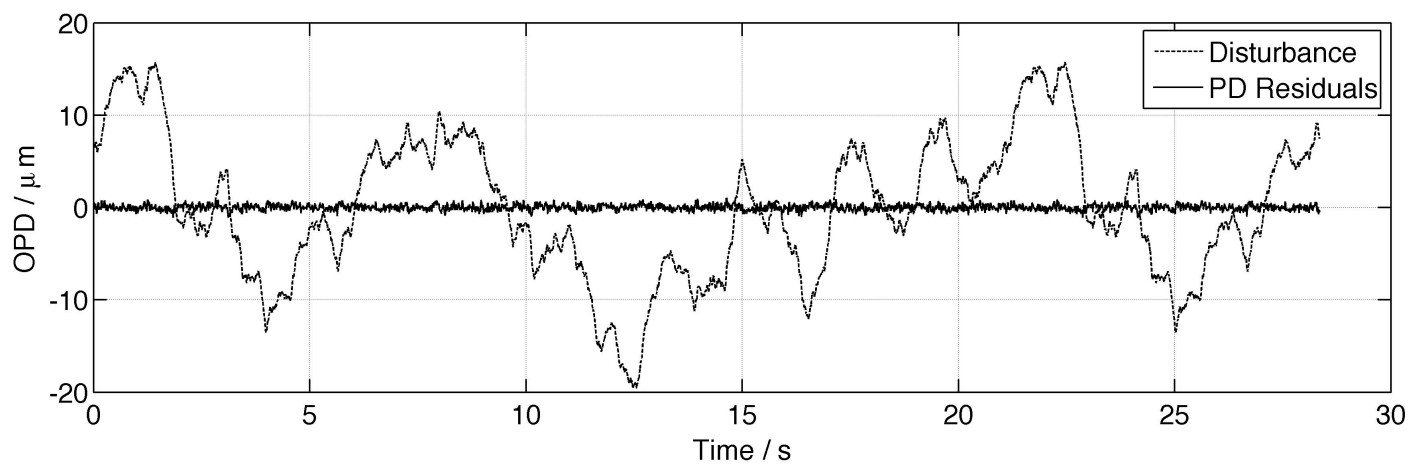

Figure 10. Atmospheric piston disturbance and the closed loop PD residuals.

The testbed was extremely useful to study and understand the FSU fringe-tracking performance. We derived a model comprising the FSU, the VLTI delay lines and the OPD controller, which reproduces the measured performance. Thus, we can compute fringe tracking residuals for a defined piston disturbance from this model. Also, we demonstrated once again that the pure delay in the control loop has to be reduced as much as possible. Increasing the OPD controller data rate from $2 \mathrm{kHz}$ to $4 \mathrm{kHz}$ does reduce the delay and significantly improves the fringe tracking performance.

Beam alignment and the corresponding injection of light into the single-mode fibres are of crucial importance for fringe tracking operation. In the case of fringe tracking with the FSU in presence of tip-tilt disturbance, the beam alignment on the fibres (optimising the overall injection) is at least as critical as the mutual fibre alignment (responsible for differential injection effects).

\section{CONCLUSIONS}

During the two years of testbed operation, a number of major and countless minor problems have been discovered in the FSU and the underlying control architecture. Fundamental improvements in terms of hardware and software were implemented and validated to solve these problems. The unstable initial beam alignment system and the unsatisfactory cold camera of the FSU were replaced and the real-time computation software was redesigned. Crucial functionalities, like the calibration procedure, were developed in the testbed, which included a major upgrade of the metrology system. In summary, the FSU evolved to a deliverable state during the testbed period.

We demonstrated robust fringe tracking with the FSU in a VLTI-similar control environment, implementing a combined group delay and phase delay tracking algorithm. In addition, the testbed was used to probe and validate novel concepts and developments like the RMN recording facility ${ }^{13}$ and an adaptive vibration cancellation algorithm for fringe tracking, ${ }^{16}$ before they were successfully implemented at the VLTI.

Most problems of the FSU have been solved and all efforts are made to solve the few remaining ones before the shipment to the VLTI observatory. With the significant knowledge and expertise acquired during the testing period, the testbed is of fundamental importance for the successful installation, commissioning and acceptance of the FSU at the observatory. This becomes even more obvious, when considering the complexity of the instrument and the limitation of testing time once the instrument is installed at the VLTI.

However, the FSU can still be improved, e.g. the real-time algorithms can be optimised in order to improve the accuracy and dynamic performance. Also, the installation and commissioning at the Paranal observatory will reveal how the FSU operation strategies have to be adapted and optimised in order to fully exploit the scientific capabilities of PRIMA. 


\section{REFERENCES}

[1] Delplancke, F., Derie, F., Lévêque, S., Ménardi, S., et al., "PRIMA for the VLTI: a status report," in [Advances in Stellar Interferometry, Proceedings of the SPIE, Volume 6268], (2006).

[2] Haguenauer, P., "Current status of the Very Large Telescope interferometer," in [These Proceedings], (2008).

[3] Launhardt, R., Henning, T. F. E., Queloz, D., and Quirrenbach, A., "The ESPRI project: narrow-angle astrometry with VLTI-PRIMA," in [These Proceedings], (2008).

[4] Bartko, H., Pfuhl, O., Eisenhauer, F., Genzel, R., et al., "Study of the science capabilities of PRIMA in the Galactic Center," in [These Proceedings], (2008).

[5] van Belle, G., Abuter, R., Delplancke, F., Ngoumou, J., and Sahlmann, J., "Extragalactic reference targets for PRIMA," in [These Proceedings], (2008).

[6] Mottini, S., Cesare, S., and Nicolini, G., "PRIMA FSU: a fringe sensor unit for the VLTI," in [Building European OLED Infrastructure. Proceedings of the SPIE, Volume 5962], (2005).

[7] Abuter, R., Rabien, S., Eisenhauer, F., Sahlmann, J., et al., "VLTI-PRIMA fringe tracking testbed," in [Advances in Stellar Interferometry. Proceedings of the SPIE, Volume 6268], (2006).

[8] Sahlmann, J., System performance characterisation of the PRIMA Fringe Sensor Unit, Master's thesis, Albert-Ludwigs-Universität, Freiburg im Breisgau (2007).

[9] Gai, M., Menardi, S., Cesare, S., Bauvir, B., et al., "The VLTI fringe sensors: FINITO and PRIMA FSU," in [New Frontiers in Stellar Interferometry. Proceedings of the SPIE, Volume 5491], (2004).

[10] Gitton, P. B., Leveque, S., Avila, G., and Phan Duc, T., "IRIS: an infrared tilt sensor for the VLTI," in [New Frontiers in Stellar Interferometry. Proceedings of SPIE Volume 5491], (2004).

[11] Bonnet, H., Bauvir, B., Wallander, A., Cantzler, M., et al., "Enabling Fringe Tracking at the VLTI," The Messenger 126 (2006).

[12] Salvadé, Y., Schuhler, N., Lévêque, S., and Le Floch, S., "High-accuracy absolute distance measurement using frequency comb referenced multiwavelength source," Applied Optics 47 (2008).

[13] Abuter, R., Popovic, D., Pozna, E., Sahlmann, J., and Eisenhauer, F., "The VLTI real-time reflective memory data streaming and recording system," in [These Proceedings], (2008).

[14] Sahlmann, J., Abuter, R., Ménardi, S., and Vasisht, G., "Astrometry with the VLTI: Calibration of the Fringe Sensor Unit for the PRIMA astrometric camera," in [Proceedings IAU Symposium No. 248], (in Press).

[15] Di Lieto, N., Sahlmann, J., Wallander, A., and Vasisht, G., "An Approach To Stabilizing Large Telescopes For Stellar Interferometry," in [Proceedings ICALEPCS 2007], (2007).

[16] Di Lieto, N., Haguenauer, P., Sahlmann, J., and Vasisht, G., "Adaptive Vibration Cancellation on Large Telescopes for Stellar Interferometry," in [These Proceedings], (2008). 